SFB/CPP-13-29, TTP13-13, LPN13-030

\title{
On the Higgs boson pair production at the LHC
}

\author{
Jonathan Grigo $^{\mathrm{a}}$, Jens Hoffa ${ }^{\mathrm{a}}$, Kirill Melnikov ${ }^{\mathrm{b}}$, Matthias Steinhauser ${ }^{\mathrm{a}}$ \\ ${ }^{a}$ Institut für Theoretische Teilchenphysik, Karlsruhe Institute of Technology (KIT), Karlsruhe, Germany \\ ${ }^{b}$ Department of Physics and Astronomy, Johns Hopkins University, Baltimore, MD, USA
}

\begin{abstract}
We compute the production cross section of a pair of Standard Model Higgs bosons at the LHC at next-to-leading order in QCD, including corrections in inverse powers of the top quark mass. We calculate these power corrections through $\mathcal{O}\left(1 / M_{t}^{8}\right)$ and study their relevance for phenomenology of the double Higgs production. We find that power corrections are significant, even for moderate values of partonic center-of-mass energies, and that convergence of the $1 / M_{t}$ expansion can be dramatically improved by factorizing the leading order cross section with full $M_{t}$-dependence.
\end{abstract}

\section{Introduction}

The recent discovery [1, 2] of a scalar particle with properties that are very similar to that of a Higgs boson completes the first stage of the quest to understand the mechanism of electroweak symmetry breaking. Indeed, after many years of preparation, ATLAS and CMS collaborations have identified an elementary particle that may be directly connected to this phenomenon. Understanding this connection will be the primary focus of particle physics in the coming years and there are two things that have to be done. First, it is important to measure couplings of the Higgs boson to gauge bosons and fermions, and to verify that the Higgs couplings to various particles are proportional to their masses. Early measurements of the couplings strengths seem to confirm this hypothesis [3]. Second, it is crucial to probe the Higgs boson self-interactions. Indeed, within the Standard Model and many of its extensions, the Higgs boson self-interaction triggers the electroweak symmetry breaking and it is important to verify that we properly understand this phenomenon.

Self-interactions of the Higgs field and the electroweak symmetry breaking induce the triple Higgs boson coupling. This coupling can be studied in the production of a pair of Higgs bosons at the LHC [4, 5, 6]. At leading order in QCD perturbation theory, the production of the Higgs boson occurs in gluon fusion and proceeds either through a box $g g \rightarrow H H$ or a triangle $g g \rightarrow H^{*}$ diagram, see Fig. 1 In the latter case the off-shell Higgs boson decays to two Higgs bosons in the final state 

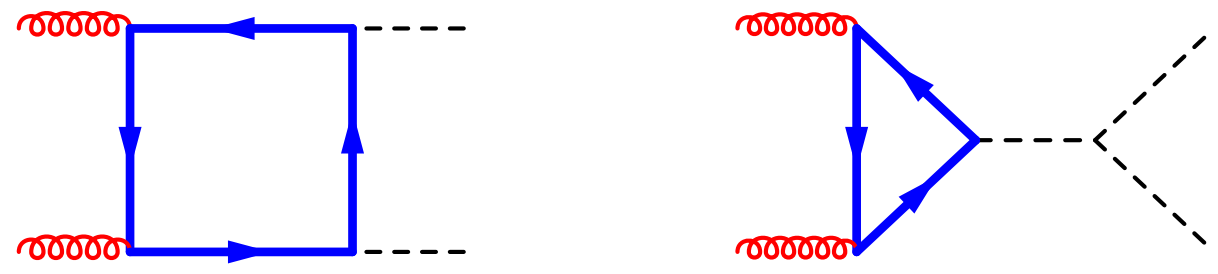

Figure 1: Box and triangle diagrams that contribute to double Higgs boson production at leading order. Solid lines refer to top quarks and dashed lines refer to Higgs bosons.

$H^{*} \rightarrow H H$, making this contribution sensitive to triple Higgs boson coupling. Unfortunately, observation of this process at the LHC is very challenging. Indeed, it has a relatively small cross section to begin with and, furthermore, it suffers from huge backgrounds that are present for almost all major decay modes of the Higgs bosons. Earlier estimates suggest that, with $600 \mathrm{fb}^{-1}$, it is possible to study the double Higgs boson production at the LHC in the $b \bar{b} \gamma \gamma$ channel [7]. More recent applications of jet substructure techniques to double Higgs production indicate that one is sensitive to this process in $b \bar{b} W^{+} W^{-}$and $b \bar{b} \tau \bar{\tau}$ channels with the integrated luminosity of $600-1000 \mathrm{fb}^{-1}[8,9]$. Since substructure techniques are still in the process of being developed, it is reasonable to expect significant improvements in these initial estimates. Therefore, we will assume an optimistic outlook about prospects for measuring the double Higgs boson production cross section at the LHC and we will try to improve the quality of theoretical description of this process within the Standard Model.

We begin by summarizing what is known about the double Higgs boson production in hadron collisions in the Standard Model. As we already mentioned, the dominant contribution to the process $p p \rightarrow H H$ is the gluon fusion that only occurs at one-loop in perturbative QCD. The corresponding partonic and hadronic cross sections were computed in Refs. [4, 5]. Part of this contribution comes from $g g \rightarrow H^{*}$ process (see Fig. 1) which is equivalent to single Higgs boson production. It is well-known that QCD radiative corrections to single Higgs production are large [10, 11, 12]. The bulk of these large radiative corrections comes from relatively soft gluons that should not be sensitive to the details of the final state as long as it is colorless. Therefore, we can expect that similar large corrections are present in case of the double Higgs boson production but, unfortunately, it is difficult to make this statement precise. This is so because computation of QCD corrections to double Higgs boson production requires four-point two-loop amplitudes with massive particles which are out of reach of contemporary technology for perturbative QCD computations.

Given this difficulty, computation of QCD corrections to double Higgs boson production was performed in the approximation where the mass of the top quark was taken to be very large compared to the partonic center-of-mass collision energy and the Higgs boson mass [13]. QCD corrections obtained in this large- $M_{t}$ approximation appear to be significant; the ratio of next-to- 
leading order cross section to leading-order cross section is close to a factor two if factorization and renormalization scales are set to $m_{H}$. However, it is unclear to what extent these large radiative corrections are relevant for phenomenology because, as was repeatedly emphasized in the literature [13, 14], the large- $M_{t}$ approximation fails to provide a good description of the $p p \rightarrow H H$ cross section for realistic values of $M_{t}, m_{H}$ and the hadronic center-of-mass collision energy. To illustrate this point, we note that the large- $M_{t}$ limit of the leading order cross section is about fifty percent smaller than the exact leading-order cross section [14].

To clarify to what extent the large radiative corrections observed in the large- $M_{t}$ limit can be trusted, we decided to compute additional terms in the $1 / M_{t}$ expansion at next-to-leading order in perturbative QCD and to explore how these power corrections affect the size of next-to-leading order (NLO) QCD effects. We note that it was also found from comparisons of cross sections at leading order 14], that accounting for additional terms in $1 / M_{t}$ expansion does not improve the agreement between expanded and exact results for moderate and high values of the partonic center-of-mass energy $\sqrt{s}$. In this situation, knowing additional terms in the expansion may not help with the phenomenology per se, but it is still useful for understanding the uncertainty that needs to be assigned to the NLO QCD prediction for $p p \rightarrow H H$. Moreover, if many terms in $1 / M_{t}$ expansion are available at next-to-leading order, we may try to improve on the quality of the expansion by factoring out the exact leading order cross section. This strategy is known to work very well in the case of single Higgs production in gluon fusion, giving us a reason to believe that it will be useful for the process $p p \rightarrow H H$ as well. With the $1 / M_{t}$ expansion at hand, we will be able to determine the range of partonic center-of-mass energies, for which this procedure works well.

The remainder of this paper is organized as follows. We explain the computational methods in Section 2 and describe numerical results in Section 3 . We present our conclusions in Section 4

\section{Computational methods}

In this Section we present technical details of our computation of the NLO QCD corrections to Higgs boson pair production in proton collisions, $p p \rightarrow H H$. The cross section is given by the product of parton distribution functions and the partonic cross section for the process $i j \rightarrow H H$, where $i$ and $j$ are the colliding partons

$$
\sigma(p p \rightarrow H H+X)=\sum_{i j} \int \mathrm{d} x_{1} \mathrm{~d} x_{2} f_{i}\left(x_{1}\right) f_{j}\left(x_{2}\right) \sigma_{i j \rightarrow H H} .
$$

At leading order in the strong coupling constant, both partons are gluons; at next-to-leading order, quark-gluon and quark-antiquark collisions start to contribute.

We take the gluon fusion channel $g g \rightarrow H H$ as an example to describe computational methods. At leading order, this process occurs through a box diagram $g g \rightarrow H H$ or through a triangle 


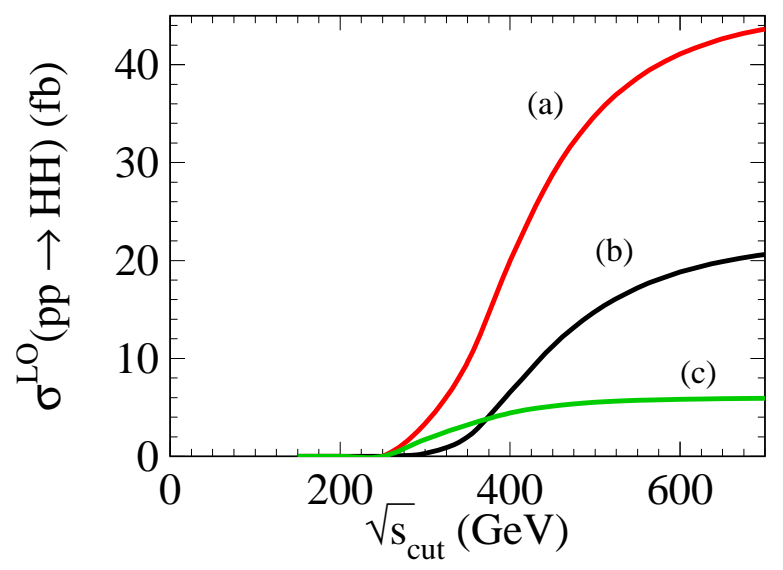

Figure 2: Leading order hadronic cross section for Higgs boson pair production at the 14 TeV LHC as the function of the upper cut on the Higgs boson pair invariant mass. Curve (b) is the full result; curve (a) is the box contribution; curve (c) is the triangle contribution. The destructive interference between box and triangle contributions is apparent. We use MSTW2008 parton distribution functions [15].

diagram $g g \rightarrow H^{*}$ with subsequent decay $H^{*} \rightarrow H H$, see Fig. 11 Both the box and the triangle diagrams are mediated by the top quark loops. Assuming that the top quark mass $M_{t}$ is much larger than both, the mass of the Higgs boson $m_{H}$ and the collision energy of the two gluons, we can understand the leading contribution to $g g \rightarrow H H$ in this large- $M_{t}$ limit using the concept of the effective Lagrangian. The effective Lagrangian for Higgs-gluon interaction can be obtained by integrating out the top quark field from the Standard Model Lagrangian. It reads [13]

$$
\mathcal{L}=\frac{\alpha_{s}}{6 \pi} \operatorname{Tr}\left[G_{\mu \nu} G^{\mu \nu}\right] \log \left(1+\frac{H}{v}\right),
$$

where $G_{\mu \nu}=G_{\mu \nu}^{a} t^{a}$ is the gluon field-strength tensor and $v$ is the vacuum expectation value of the Higgs field. Expanding Eq. (2) to second order in $H / v$, we obtain terms that lead to $g g H$ and $g g H H$ interactions. The scattering amplitude for $g\left(p_{1}\right) g\left(p_{2}\right) \rightarrow H H$, that follows from the effective Lagrangian Eq. (2), reads

$$
\mathcal{A}_{\lambda_{1} \lambda_{2}}=\frac{\alpha_{s} \delta_{a b}}{8 \pi v} \delta_{\lambda_{1} \lambda_{2}}\left[-\frac{4}{3}+\frac{4 m_{H}^{2}}{s-m_{H}^{2}}\right],
$$

where $s=2 p_{1} \cdot p_{2}, \lambda_{1,2}$ are helicities of the two gluons and $a, b$ are their color indices. We note that the first term in square brackets in Eq. (3) comes from the box diagram and the second term from the triangle diagram, that contains a triple Higgs coupling. It is interesting to note that box and triangle contributions to $g g \rightarrow H H$ amplitude tend to strongly cancel each other. For example, at the partonic threshold $s=4 m_{H}^{2}$, the cancellation between the two contributions is exact. To further illustrate this point, in Fig. 2 we show the leading order Higgs boson pair production cross section at the $14 \mathrm{TeV}$ LHC in dependence of the upper cut on the partonic center-of-mass collision energy. As can be seen from that Figure, the impact of the triple Higgs boson coupling 
on the observable cross section comes from the destructive interference of the box and triangle contributions. It reduces the cross section by almost a factor of two for a broad range of $\sqrt{s_{\text {cut }}}$ and implies linear sensitivity of the $p p \rightarrow H H$ production cross section to small changes in triple Higgs boson coupling. It also follows from Fig. 2 that the $p p \rightarrow H H$ cross section gets saturated at values of the Higgs pair invariant mass cut $\sqrt{s_{\text {cut }}} \approx 600 \sim 700 \mathrm{GeV}$. Therefore, for a reliable NLO computation, we require accurate description of partonic cross sections for double Higgs boson production up to $\sqrt{s} \sim 600 \mathrm{GeV}$ since higher partonic center-of-mass energies are not important for the hadronic $p p \rightarrow H H$ cross section.

While Eq. (3) is useful for thinking about Higgs boson production, it does not lead to an adequate description of $p p \rightarrow H H$ cross section since further terms in $1 / M_{t}$ expansion are needed. Deriving such terms is straightforward at leading order in perturbative QCD where one can simply obtain an exact result by computing the required one-loop diagrams. Unfortunately, it becomes prohibitively difficult to do that at next-to-leading order where computation of two-loop box diagrams for $g g \rightarrow H H$ with full $M_{t}$-dependence is required. To overcome this problem, we expand the relevant NLO cross sections in powers of $1 / M_{t}$. We do so by employing a procedure that has been used for the computation of finite- $M_{t}$ corrections to the production cross section of a single Higgs boson in gluon fusion [16]. In what follows we briefly describe it.

We start by representing the total cross section for the double Higgs boson production by the properly-normalized discontinuity of the gluon forward scattering amplitudes (see Fig. 3 for sample Feynman diagrams),

$$
\sigma(g g \rightarrow H H) \sim \operatorname{Disc}\left[\mathcal{A}_{g g \rightarrow g g}\right] .
$$

The discontinuity is obtained by cutting diagrams that contribute to forward-scattering amplitudes through two Higgs lines at leading and next-to-leading order, and through two Higgs lines and a gluon line at next-to-leading order. As explained in Ref. [17], Eq. (4) is helpful because the procedure of taking the discontinuity of the forward scattering amplitude $\mathcal{A}_{g g \rightarrow g g}$ commutes with modern techniques that can be used to compute it. Indeed, to calculate diagrams that contribute to $\mathcal{A}_{g g \rightarrow g g}$, we first perform the large- $M_{t}$ expansion by treating masses and momenta of the Higgs bosons and, where appropriate, of a cut gluon line, to be much smaller than the top quark mass. Once this expansion is carried out, we obtain a large number of Feynman integrals and we reduce them to a minimal set of master integrals using the integration-by-parts technique [18, 19]. The number of master integrals is small - we require one integral for leading and seven integrals for next-to-leading order computation. The calculation proceeds through a sequence of computer-algebra programs developed for computing mass corrections to single Higgs boson production in gluon fusion [16]. Relevant three- and four-loop diagrams that contribute to the forward scattering amplitude are obtained with QGRAF [20], supplemented with additional scripts to remove unnecessary diagrams. Each diagram is then expanded assuming $M_{t}^{2} \gg m_{H}^{2}$ and 

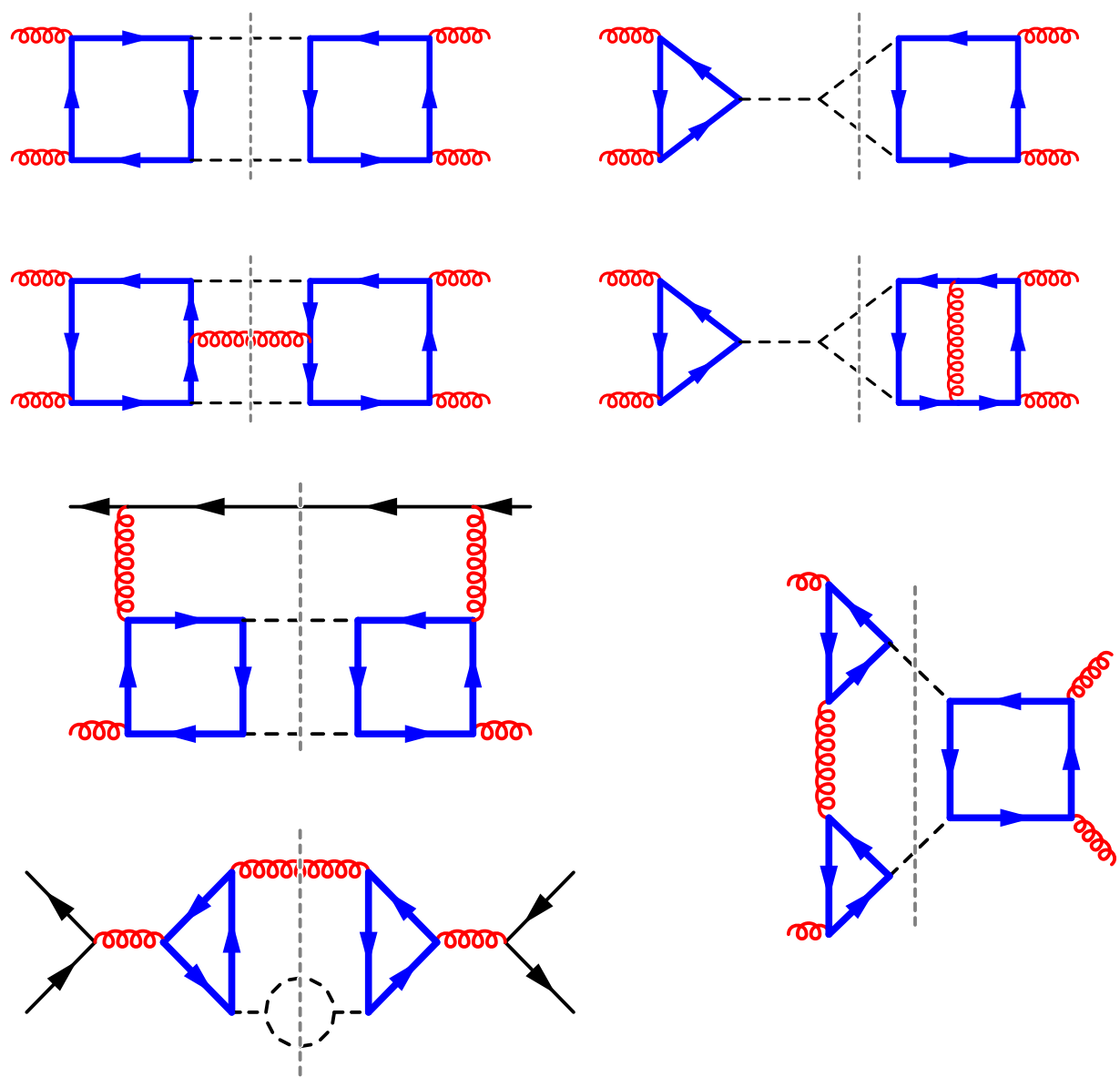

Figure 3: Examples of forward scattering amplitudes that we need to consider. Dashed vertical lines represent unitarity cuts. Solid lines are top and light quarks, dashed lines are Higgs bosons.

$M_{t}^{2} \gg s$ using programs q2e and exp 21]. We express each Feynman diagram as linear combination of scalar integrals using FORM 22 and its parallel version TFORM 23]. The reduction to master integrals is performed using FIRE [24, 25] 1 which implements the Laporta algorithm [26]. The first two expansion terms in $\rho$ are computed for general QCD gauge parameter $\xi$ and the independence of the final result of $\xi$ is used as a check of the computation. Furthermore, our result for the $\rho^{0}$ contribution to the partonic cross section agrees with Ref. [13].

Sample master integrals that appear at NLO are shown in Fig. 4. We will discuss their computation in what follows. Note that we require these master integrals to higher orders in the dimensional-regularization parameter $\epsilon=(d-4) / 2$ since they multiply the divergent reduction coefficients. In principle, it is possible to derive exact expressions for these integrals but this is cumbersome. However, it is very easy to construct an expansion of these integrals around the double Higgs partonic threshold to, essentially, arbitrary order in the expansion parameter. We

\footnotetext{
${ }^{1}$ We thank A.V. Smirnov and V.A. Smirnov for allowing us to use the unpublished C++ version of FIRE.
} 

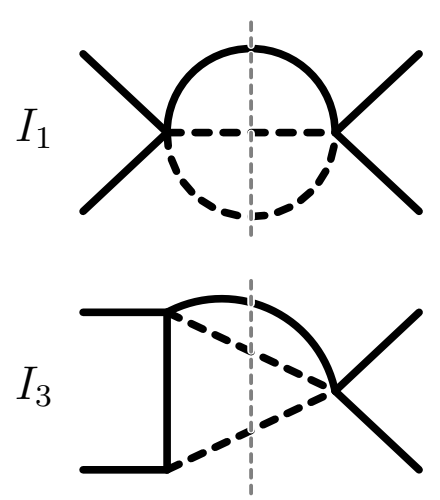

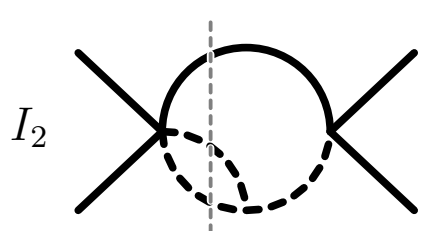

$I_{4}$

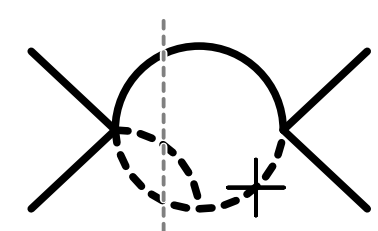

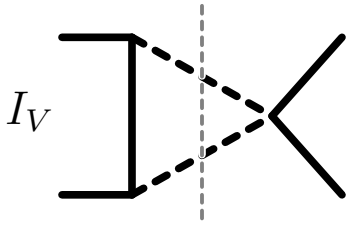

Figure 4: The master integrals $I_{1}, I_{2}, I_{3}, I_{4}$ and $I_{V}$. Dashed lines cut through propagators that are replaced by the mass-shell conditions. Dashed lines are the Higgs boson propagators and solid lines correspond to massless scalar propagators. In the case of $I_{4}$ the cross indicates a propagator raised to power minus one. $I_{V}$ contributes to the virtual corrections at next-to-leading order.

now show how this is done for individual master integrals.

For the leading order process $g\left(p_{1}\right)+g\left(p_{2}\right) \rightarrow H\left(p_{3}\right)+H\left(p_{4}\right)$, there is just one master integral which corresponds to the available phase space for final state particles

$$
I_{0}=\int\left[\mathrm{d} p_{3}\right]\left[\mathrm{d} p_{4}\right](2 \pi)^{d} \delta^{(d)}\left(p_{1}+p_{2}-p_{3}-p_{4}\right)
$$

where $[\mathrm{d} p]=\mathrm{d}^{d-1} p /\left((2 \pi)^{(d-1)} 2 p_{0}\right), p_{1}^{2}=p_{2}^{2}=0$ and $p_{3}^{2}=p_{4}^{2}=m_{H}^{2}$. At next-to-leading order, several different types of master integrals appear since $\mathcal{O}\left(\alpha_{s}\right)$ corrections can either come from real gluon emissions or from virtual gluon exchanges. For the real emission corrections, four master integrals appear as the result of the reduction but only three of them are linearly independent. For the virtual corrections, we need two-loop vacuum bubble integrals and one additional master integral that we describe below.

The four master integrals that we require to compute for the real emission corrections are (see Fig. 4)

$$
\begin{aligned}
I_{1} & =\int\left[\mathrm{d} p_{3}\right]\left[\mathrm{d} p_{4}\right]\left[\mathrm{d} p_{5}\right](2 \pi)^{d} \delta^{(d)}\left(p_{1}+p_{2}-p_{3}-p_{4}-p_{5}\right), \\
I_{2} & =\int\left[\mathrm{d} p_{3}\right]\left[\mathrm{d} p_{4}\right]\left[\mathrm{d} p_{5}\right](2 \pi)^{d} \delta^{(d)}\left(p_{1}+p_{2}-p_{3}-p_{4}-p_{5}\right) \times\left(\left(p_{3}+p_{4}\right)^{2}-m_{H}^{2}\right), \\
I_{3} & =\int\left[\mathrm{d} p_{3}\right]\left[\mathrm{d} p_{4}\right]\left[\mathrm{d} p_{5}\right](2 \pi)^{d} \delta^{(d)}\left(p_{1}+p_{2}-p_{3}-p_{4}-p_{5}\right) \times\left(p_{2}-p_{4}\right)^{2}, \\
I_{4} & =\int\left[\mathrm{d} p_{3}\right]\left[\mathrm{d} p_{4}\right]\left[\mathrm{d} p_{5}\right](2 \pi)^{d} \delta^{(d)}\left(p_{1}+p_{2}-p_{3}-p_{4}-p_{5}\right) \times\left(\left(p_{3}+p_{4}\right)^{2}-m_{H}^{2}\right)^{-1} .
\end{aligned}
$$

To compute $I_{1}$, it is convenient to introduce integration over total momentum of the two Higgs bosons $Q=p_{3}+p_{4}$ by inserting $\mathrm{d}^{d} Q \delta^{(d)}\left(Q-p_{3}-p_{4}\right)=1$ into the integrand of $I_{1}$. We obtain

$$
I_{1}=\int_{4 m_{H}^{2}}^{s} \frac{\mathrm{d} Q^{2}}{(2 \pi)} \operatorname{Lips}\left(Q, p_{3}, p_{4}\right) \operatorname{Lips}\left(P_{12}, Q, p_{5}\right)
$$


where $P_{12}=p_{1}+p_{2}$ and Lips is the two-particle Lorentz-invariant phase space defined as

$$
\operatorname{Lips}(p, q, r)=\int[\mathrm{d} q][\mathrm{d} r](2 \pi)^{d} \delta^{(d)}(p-q-r) .
$$

It is straightforward to compute the phase spaces that appear in Eq. (77). We find

$$
\begin{aligned}
& \operatorname{Lips}\left(Q, p_{3}, p_{4}\right)=Q^{-2 \epsilon} \frac{\Omega_{d-1} 2^{2 \epsilon}}{(2 \pi)^{d-2} 8}\left(1-\frac{4 m_{H}^{2}}{Q^{2}}\right)^{1 / 2-\epsilon}, \\
& \operatorname{Lips}\left(P_{12}, Q, p_{5}\right)=s^{-\epsilon} \frac{\Omega_{d-1} 2^{2 \epsilon}}{(2 \pi)^{d-1} 8}\left(1-\frac{Q^{2}}{s}\right)^{1-2 \epsilon},
\end{aligned}
$$

where $s=P_{12}^{2}$ and $\Omega_{d}=2 \pi^{d / 2} / \Gamma(d / 2)$ is the solid angle of the $d$-dimensional space. Combining the above formulas, we obtain

$$
I_{1}=\mathcal{N} s^{-\epsilon} \int_{4 m_{H}^{2}}^{s} \mathrm{~d} Q^{2} Q^{-2 \epsilon}\left(1-\frac{4 m_{H}^{2}}{Q^{2}}\right)^{1 / 2-\epsilon}\left(1-\frac{Q^{2}}{s}\right)^{1-2 \epsilon},
$$

where the normalization factor $\mathcal{N}$ reads

$$
\mathcal{N}=\left[\frac{\Gamma(1+\epsilon)}{(4 \pi)^{d / 2}}\right]^{2}\left[1+4 \epsilon+\left(12-\frac{2 \pi^{2}}{3}\right) \epsilon^{2}+\mathcal{O}\left(\epsilon^{3}\right)\right] .
$$

To facilitate the computation of the integral, we change the integration variable by writing $Q^{2}=$ $s(1-\delta \mu)$ and express the result in terms of the variable $\delta=1-4 m_{H}^{2} / s$. We obtain

$$
I_{1}=\mathcal{N} s^{1-2 \epsilon} \delta^{5 / 2-3 \epsilon} \int_{0}^{1} \frac{\mathrm{d} \mu}{\sqrt{1-\delta \mu}}(1-\mu)^{1 / 2-\epsilon} \mu^{1-2 \epsilon} .
$$

The integrand of $I_{1}$ can now be expanded in a Taylor series in $\delta$ and the resulting integrals can be evaluated in a straightforward way.

We now discuss the evaluation of $I_{2}$. Note that the difference between $I_{2}$ and $I_{1}$ is due to additional terms in the integrand that, however, only depend on $Q^{2}$ since $\left(p_{3}+p_{4}\right)^{2}-m_{H}^{2}=$ $Q^{2}-m_{H}^{2}$. Therefore, the integral representation for $I_{2}$ can be easily deduced from Eq. (10). We find

$$
I_{2}=\mathcal{N} s^{-\epsilon} \int_{4 m_{H}^{2}}^{s} \mathrm{~d} Q^{2} Q^{-2 \epsilon}\left(1-\frac{4 m_{H}^{2}}{Q^{2}}\right)^{1 / 2-\epsilon}\left(1-\frac{Q^{2}}{s}\right)^{1-2 \epsilon} \times\left(Q^{2}-m_{H}^{2}\right) .
$$

Trading $Q^{2}$ for $\mu$, we obtain

$$
I_{2}=\mathcal{N} s^{2-2 \epsilon} \delta^{5 / 2-3 \epsilon} \int_{0}^{1} \frac{\mathrm{d} \mu}{\sqrt{1-\delta \mu}}(1-\mu)^{1 / 2-\epsilon} \mu^{1-2 \epsilon}\left[\frac{3}{4}+\delta\left(\frac{1}{4}-\mu\right)\right],
$$

and the Taylor expansion in $\delta$ becomes straightforward.

As the next step, we show that the integral $I_{3}$ is a linear combination of $I_{1}$ and $I_{2}$. Indeed, since

$$
I_{3}=\int\left[\mathrm{d} p_{3}\right]\left[\mathrm{d} p_{4}\right]\left[\mathrm{d} p_{5}\right](2 \pi)^{d} \delta^{(d)}\left(p_{1}+p_{2}-p_{3}-p_{4}-p_{5}\right)\left(m_{H}^{2}-2 p_{2} \cdot p_{4}\right),
$$


we can simplify it by using the following equations

$$
\int\left[\mathrm{d} p_{3}\right]\left[\mathrm{d} p_{4}\right](2 \pi)^{d} \delta^{(d)}\left(Q-p_{3}-p_{4}\right)\left(m_{H}^{2}-2 p_{2} \cdot p_{4}\right)=\left(m_{H}^{2}-p_{2} \cdot Q\right) \operatorname{Lips}\left(Q, p_{3}, p_{4}\right),
$$

and

$$
\begin{aligned}
& \int\left[\mathrm{d} p_{5}\right][\mathrm{d} Q](2 \pi)^{d} \delta^{(d)}\left(P_{12}-p_{5}-Q\right)\left(m_{H}^{2}-p_{2} \cdot Q\right) \\
& =\int\left[\mathrm{d} p_{5}\right][\mathrm{d} Q](2 \pi)^{d} \delta^{(d)}\left(P_{12}-p_{5}-Q\right)\left(m_{H}^{2}-\frac{p_{2} \cdot P_{12}\left(s+Q^{2}\right)}{2 s}\right) \\
& =\left(m_{H}^{2}-\frac{s+Q^{2}}{4}\right) \operatorname{Lips}\left(P_{12}, Q, p_{5}\right) .
\end{aligned}
$$

Therefore, $I_{3}$ becomes

$$
I_{3}=\mathcal{N} s^{-\epsilon} \int_{4 m_{H}^{2}}^{Q^{2}} \mathrm{~d} Q^{2} Q^{-2 \epsilon}\left(1-\frac{4 m_{H}^{2}}{Q^{2}}\right)^{1 / 2-\epsilon}\left(1-\frac{Q^{2}}{s}\right)^{1-2 \epsilon}\left(m_{H}^{2}-\frac{s+Q^{2}}{4}\right) .
$$

Using Eqs. (10) and (13), it is easy to see that $I_{3}$ can be represented as a linear combination of $I_{1}$ and $I_{2}$

$$
I_{3}=\left(m_{H}^{2}-\frac{s}{4}\right) I_{1}-\frac{1}{4}\left(I_{2}+m_{H}^{2} I_{1}\right)
$$

Finally, the integrand for $I_{4}$ differs from that for $I_{2}$ because the term $\left(Q^{2}-m_{H}^{2}\right)$ occurs in the denominator instead of the numerator; therefore, the useful representation for $I_{4}$ can be easily derived following the steps described above. We obtain

$$
I_{4}=4 \mathcal{N} s^{-2 \epsilon} \delta^{5 / 2-3 \epsilon} \int_{0}^{1} \frac{\mathrm{d} \mu}{\sqrt{1-\delta \mu}} \frac{(1-\mu)^{1 / 2-\epsilon} \mu^{1-2 \epsilon}}{3+\delta(1-4 \mu)},
$$

and we can expand it in $\delta$ in a straightforward way.

Finally, additional master integrals are needed for the virtual corrections. The virtual corrections required for this are the two-loop ones and their large-mass expansion leads to two distinct contributions. The first contribution arises when the loop momenta are comparable to the mass of the top quark. In this case, the two-loop diagrams are expandable in external momenta and the Higgs boson masses. Using the integration-by-parts, the two-loop integrals are mapped onto a single two-loop vacuum bubble integral. In a situation where one loop-momentum is comparable to the top mass and the other loop-momentum is comparable to the Higgs boson mass or to the partonic collision energy, a two-loop diagram factorizes into a product of one-loop diagrams and we do not discuss it here. In addition, at next-to-leading order diagrams appear where two Higgs bosons are produced in a way that involves exchange of a gluon in a $t$-channel, see Fig. 3 , The computation of these diagrams requires a new master integral. It reads (see Fig. (4)

$$
I_{V}=\int\left[\mathrm{d} p_{3}\right]\left[\mathrm{d} p_{4}\right](2 \pi)^{d} \delta\left(p_{1}+p_{2}-p_{3}-p_{4}\right) \times\left(p_{1}-p_{3}\right)^{-2}
$$


We calculate it following the above discussion and obtain

$$
I_{V}=-\mathcal{N}_{V} 8 \pi s^{-1-\epsilon} \delta^{1 / 2-\epsilon} \int_{0}^{1} \mathrm{~d} x \frac{x^{-\epsilon}(1-x)^{-\epsilon}(1+\delta)}{(1+\delta)^{2}-4 \delta(1-2 x)^{2}},
$$

where

$$
\mathcal{N}_{V}=\frac{\Gamma(1+\epsilon)}{(4 \pi)^{d / 2}}\left(1-\frac{\pi^{2}}{6} \epsilon^{2}+\mathcal{O}\left(\epsilon^{3}\right)\right) .
$$

Expanding $I_{V}$ in $\delta$ is straightforward.

Once the reduction of the contributing diagrams to master integrals is performed and once explicit expressions for master integrals are substituted, we obtain unrenormalized results for partonic cross sections. To obtain physical results, we need to renormalize the strong coupling constant and the top quark mass to remove the ultraviolet divergences, and to renormalize parton distribution functions to remove the collinear singularities associated with gluon emissions from the initial state. For the ultraviolet renormalization, we use the one-loop expressions

$$
\alpha_{s}^{\text {bare }}=\frac{\mu^{2 \epsilon} \alpha_{s}}{S_{\epsilon}}\left(1-\frac{\beta_{0}}{\epsilon} \frac{\alpha_{s}}{2 \pi}+\cdots\right), \quad M_{t}^{\text {bare }}=M_{t}\left[1-\frac{C_{F} \alpha_{s}}{\pi S_{\epsilon}}\left(\frac{3}{4 \epsilon}-1-\frac{3}{4} \ln \frac{\mu^{2}}{M_{t}^{2}}\right)+\cdots\right] .
$$

Here, $S_{\epsilon}=(4 \pi)^{-\epsilon} e^{\gamma \epsilon}, \gamma=0.5772 \ldots$ is the Euler constant, $\alpha_{s} \equiv \alpha_{s}(\mu)$ is the $\overline{\mathrm{MS}}$ QCD coupling constant evaluated at the scale $\mu, \beta_{0}=11 N_{c} / 6-n_{f} / 3$ is the one-loop QCD beta-function and $M_{t}$ is the pole top quark mass. For consistent ultraviolet renormalization, we first use $n_{f}=6$ but express our final result for cross section through $\alpha_{s}$ with five active flavours by using the one-loop decoupling relation for the top quark. Ellipses in Eq. (24) stand for contributions suppressed by additional powers of $\alpha_{s}$.

The collinear renormalization is performed in the standard way, by redefining parton distribution functions. The corresponding modification of the NLO cross sections, required to make it finite, reads

$$
\sigma_{g g}^{(1)}(s)=\delta \bar{\sigma}_{g g}^{(1)}(s)+\frac{1}{\epsilon} \int_{x_{\min }}^{1} \mathrm{~d} x P_{g g}(x) \sigma_{g g}^{(0)}(x s),
$$

where $x_{\min }=4 m_{H}^{2} / s$ is determined from the Higgs pair production threshold in $g g$ collisions and $P_{g g}$ is the gluon splitting function

$$
P_{g g}=C_{A}\left[\frac{1}{(1-x)_{+}}-1+\frac{1-x}{x}+x(1-x)\right]+\delta(1-x)\left(\frac{11 C_{A}}{12}-\frac{n_{f}}{6}\right),
$$

with $C_{A}=3$ for QCD. Similar equations hold also for $q g, \bar{q} g$ and $q \bar{q}$ production channels; since they are standard, we do not present them here.

\section{Numerical results}

In this Section we present our results for the NLO QCD corrections to the double Higgs boson production cross section at the LHC. Values of the Higgs boson mass and the on-shell top quark 

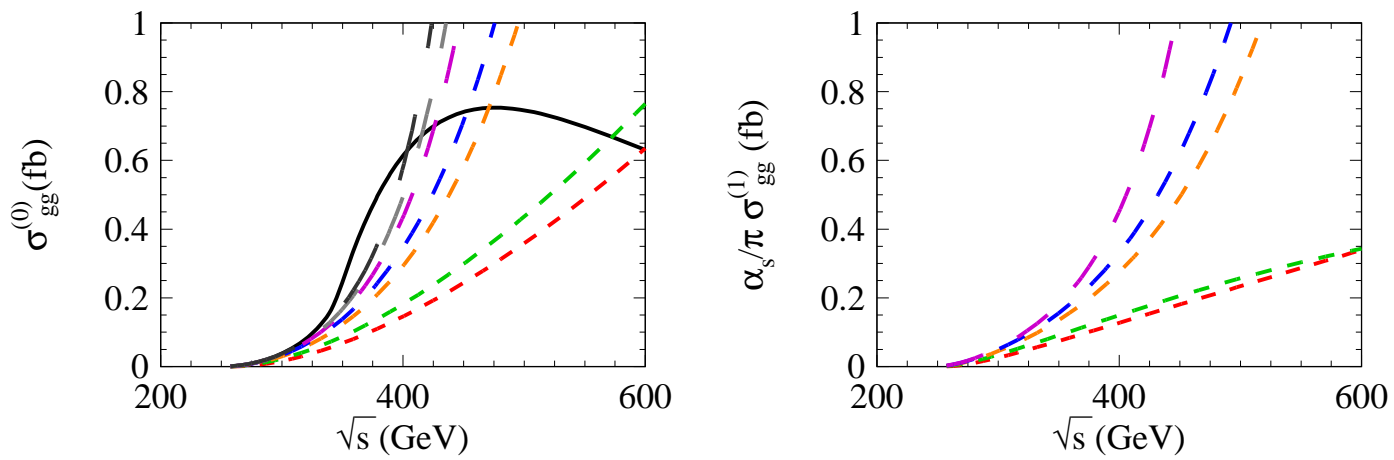

Figure 5: Leading order partonic $g g \rightarrow H H$ cross section (left pane) and next-to-leading order contribution to $g g \rightarrow H H$ cross section $\alpha_{s} / \pi \times \sigma_{g g}^{(1)}$ (right pane), in fb. Different lines correspond to 1) exact leading order cross section - black solid; 2) cross sections expanded to $\mathcal{O}\left(\rho^{0}\right)$ - short-dashed red; to $\mathcal{O}\left(\rho^{1}\right)$ - short-dashed green; to $\mathcal{O}\left(\rho^{2}\right)$ - dashed orange; to $\mathcal{O}\left(\rho^{3}\right)$ - dashed blue; to $\mathcal{O}\left(\rho^{4}\right)$ - dashed violet; to $\mathcal{O}\left(\rho^{5}\right)$ - long-dashed light gray; to $\mathcal{O}\left(\rho^{6}\right)$ - long-dashed dark gray; See text for the description of input parameters.

mass are taken to be $m_{H}=126 \mathrm{GeV}$ [1, 2] and $M_{t}=173.18 \mathrm{GeV}$ [27], respectively. We begin with discussing partonic cross sections at leading and next-to-leading order. It is convenient to express the cross sections using two variables, $x=4 m_{H}^{2} / s=1-\delta$ and $\rho=m_{H}^{2} / M_{t}^{2}$. As we explained in the previous Section, we compute the $H H$ production cross section as series in $\rho=m_{H}^{2} / M_{t}^{2}$. The expansion starts at $\rho^{0}$ and we are able to obtain five terms of the $\rho$-expansion, up to $\mathcal{O}\left(\rho^{4}\right)$, for the $g g$ partonic channel and seven terms, up to $\mathcal{O}\left(\rho^{6}\right)$, for the $q g$ and $q \bar{q}$ channel. The master integrals are computed as an expansion in the parameter $\delta=1-x$; for the final results all terms up to $\mathcal{O}\left(\delta^{50}\right)$ are included. The partonic cross sections are defined as

$$
\sigma_{i j \rightarrow H+X}(s, \rho)=\delta_{i g} \delta_{j g} \sigma_{g g}^{(0)}(s, \rho)+\frac{\alpha_{s}}{\pi} \sigma_{i j}^{(1)}(s, \rho)
$$

where $\sigma_{i j}^{(1)}$ is the $\mathcal{O}\left(\alpha_{s}\right)$ correction to the leading order cross section. For the discussion of the partonic cross sections, we set the factorization and the renormalization scales to $\mu_{r}=\mu_{f}=2 m_{H}$. We will describe the scale dependence of our results below when we consider the hadronic cross section.

We begin by showing some results for the $g g$ channel. In Fig. [5 we compare $\sigma_{g g}^{(0)}(s, \rho)$ with seven approximate cross sections that are obtained by expanding $\sigma_{g g}^{(0)}$ in $\rho$ through $\mathcal{O}\left(\rho^{i}\right), i=$ $0, \ldots, 6$. It is clear from Fig. 5 that the convergence of the expansion is poor. Indeed, already at $\sqrt{s} \sim 350 \mathrm{GeV}$, there is a sizable difference between the exact and the expanded result. In the right pane of Fig. 5 we show the NLO contribution to the cross section expanded to different orders in $\rho$. Similar to the leading order case, the $1 / M_{t}$ expansion does not appear to converge.

The bad convergence of the $1 / M_{t}$ expansion should not be very surprising. Indeed, we note that the expansion is not supposed to work beyond, or even close to, the top quark threshold that occurs at $\sqrt{s}=2 M_{t}$. Therefore, using the expansion techniques described above, we can only hope 

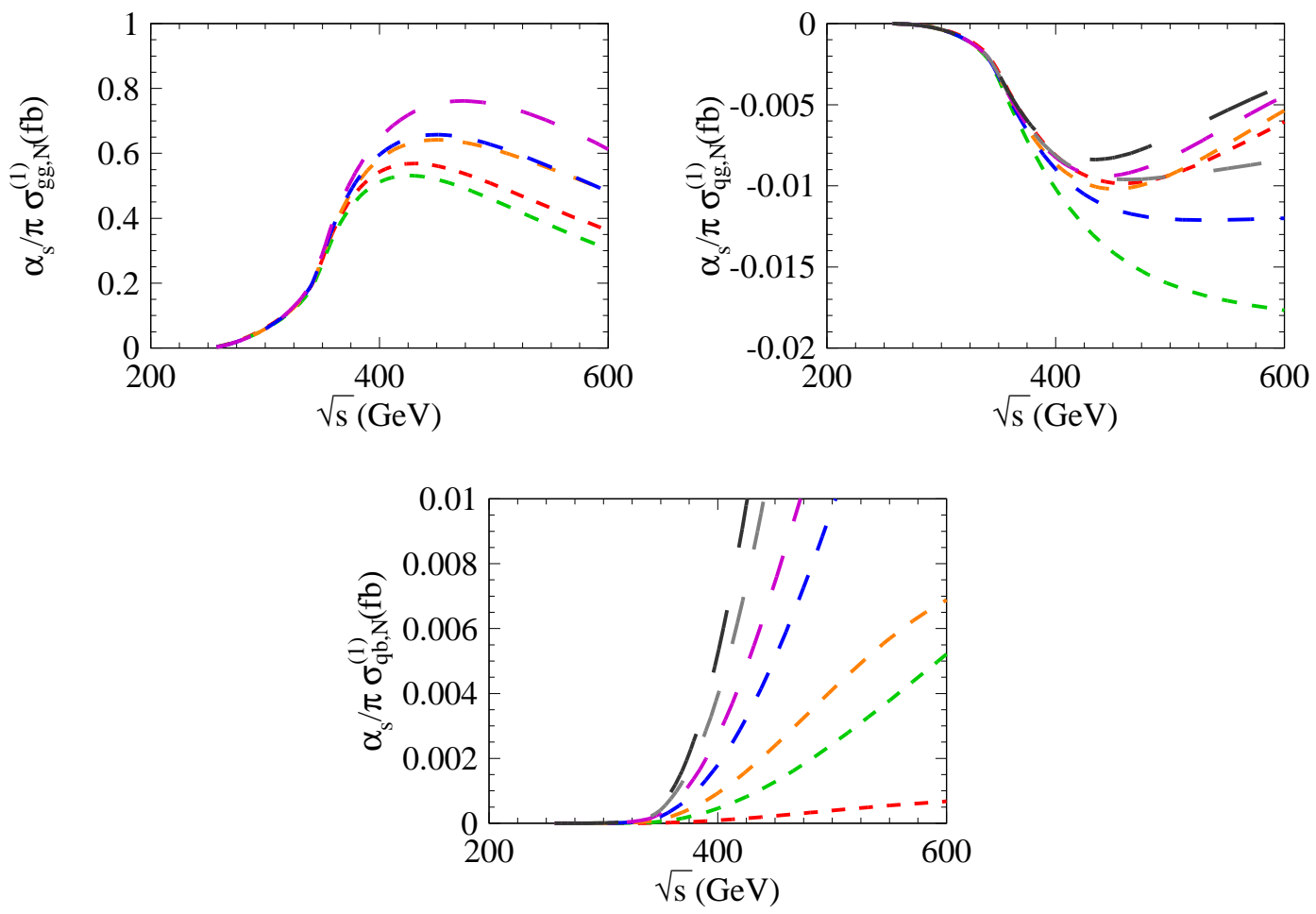

Figure 6: Next-to-leading order contribution to $g g \rightarrow H H, q g \rightarrow H H$ and $q \bar{q} \rightarrow H H$ cross sections re-scaled by exact leading order result, in fb. The color coding is as in Fig. 5

to obtain reliable results for values of $s \geq 4 M_{t}^{2}$ if we can show that corrections do not strongly depend on $s$. From this perspective, the situation is similar to what occurs in the single Higgs boson production in gluon fusion where the applicability of radiative corrections computed in the large- $M_{t}$ approximation is usually extended by combining them with the exact leading order cross section for $g g \rightarrow H$. The validity of such an approach in single Higgs production is verified by comparing it to the exact results at NLO 12 and by its consistency with known power corrections to the large- $M_{t}$ limit at NNLO QCD [28, 29, 16, 30, 31]. Motivated by the success of this approach to QCD corrections in single Higgs boson production, we apply it to Higgs pair production as well. We write the NLO QCD contribution to the partonic cross section as

$$
\sigma_{i j, N}^{(1)}=\sigma_{g g, \text { exact }}^{(0)} \Delta_{i j}^{(N)}, \quad \Delta_{i j}^{(N)}=\frac{\sigma_{i j, \exp }^{(1)}}{\sigma_{g g, \exp }^{(0)}}=\frac{\sum_{n=0}^{N} c_{i j, n}^{\mathrm{NLO}} \rho^{n}}{\sum_{n=0}^{N} c_{g g, n}^{\mathrm{LO}} \rho^{n}},
$$

where both numerator and denominator of the $\Delta$-factor are expanded to the same order in $\rho$. By changing $N$ in the above formula, we can check the stability of our computation against additional power corrections. Ideally, $\Delta_{i j}^{(N)}$, should become $N$-independent, after sufficient number of terms are included in the numerator and denominator in Eq. (28). 

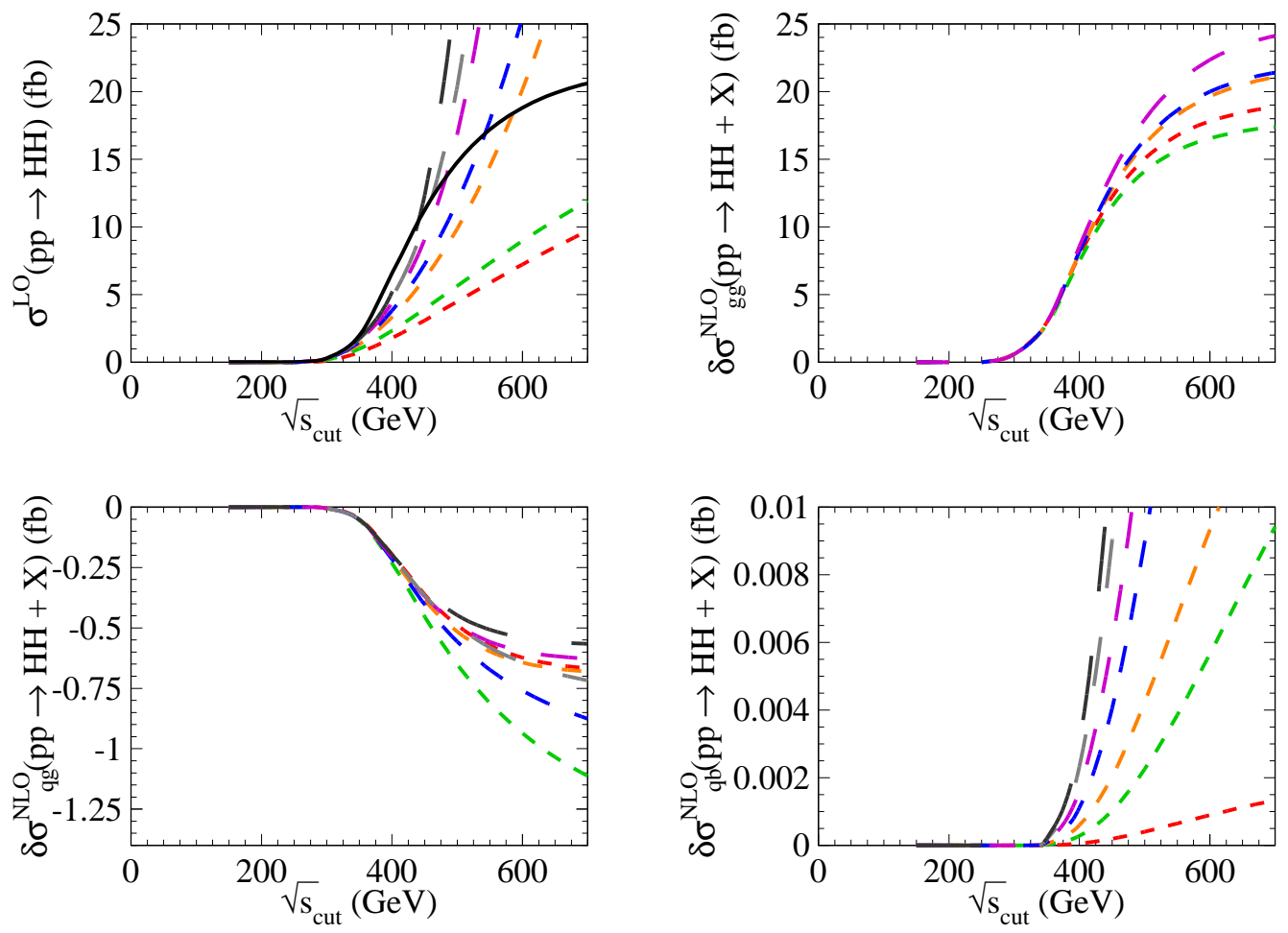

Figure 7: Leading and next-to-leading order contributions to hadronic production cross sections of Higgs boson pairs. Different curves represent the cross section obtained through expansion to different orders in $\rho$; rescaling by the leading order cross section is always included. The color coding is as in Fig. 5

Our results for $\sigma_{g g, N}^{(1)}$ are shown in Fig. 6. We see that for $N=0,1,2,3,4, \sigma_{g g, N}^{(1)}$ is fairly stable over a broad range of partonic center-of-mass energies. In fact, there is practically no difference between $\sigma_{g g, 2}^{(1)}$ and $\sigma_{g g, 3}^{(1)}$ all the way up to $\sqrt{s} \sim 600 \mathrm{GeV}$ but, unfortunately, $\sigma_{g g, 4}^{(1)}$ shows a 13 percent increase relative to $\sigma_{g g, 3}^{(1)}$ for $\sqrt{s} \sim 450 \mathrm{GeV}$. The situation is similar for the $q g$ channel and it is much worse for $q \bar{q}$ channel where even with as many as seven terms of the $1 / M_{t}$ expansion the convergence seems poor, see Fig. 6. To understand the differences between partonic channels, we note that the leading order cross section $\sigma_{g g}^{(0)}$ does not properly describe the kinematic features of $\sigma_{q g}^{(1)}$ and $\sigma_{q \bar{q}}^{(1)}$. Indeed, the $q g$ scattering, for example, mainly occurs through a $t$-channel gluon exchange which, for large $s$, has little to do with the leading order process of a gluon fusion into a pair of Higgs bosons. Fortunately, these subtleties do not impact predictions for hadronic cross sections because the $q g$ and $q \bar{q}$ channels are numerically small - they provide at most ten percent correction to the next-to-leading order contribution to Higgs boson pair production cross section. Therefore, even relatively low accuracy for $q g$ and $q \bar{q}$ channels is sufficient for reliable phenomenology.

We proceed now to the discussion of hadronic cross sections. To obtain numerical results shown below we employ MSTW2008 parton distribution functions (PDFs) [15]. We consistently 
use leading order PDFs to compute leading order cross sections and next-to-leading order PDFs to compute next-to-leading order cross sections. We assume the energy of the LHC to be $14 \mathrm{TeV}$. Values of the strong coupling constants $\alpha_{s}\left(M_{\mathrm{Z}}\right)$ that we use in our computation are obtained from the MSTW PDF fit. Their leading and next-to-leading order values are 0.139384 and 0.120176 , respectively.

We present results for hadronic cross sections as a function of the upper cut on the partonic center-of-mass energy $\sqrt{s_{\text {cut }}}$. This allows us to explore the stability of the $1 / M_{t}$ expansion of the Higgs boson pair production cross section as we move from the threshold region, where this expansion works well, to the high-energy region where this expansion becomes less reliable. We can also use this cut as a proxy for the Higgs boson pair invariant mass cut which may be useful for enhancing observability of the triple Higgs boson coupling. We note that at leading order in $\mathrm{QCD}$, a cut on partonic center of mass energy is equivalent to the cut on the invariant mass of the Higgs boson pair while at next-to-leading order the equivalence is not exact anymore. For all results below, we use the NLO contributions to partonic cross sections re-scaled with exact leading order cross section, cf. Eq. (28).

Our results for leading and next-to-leading order contributions to hadronic cross sections are shown in Fig. 7. The NLO QCD predictions for hadronic cross sections appear to be more reliable than similar results for partonic cross sections; the reason for this is the known enhancement of gluon luminosity at small values of $s$ which reduces contributions of large- $s$ partonic processes to hadronic cross sections. To illustrate this point, we note that even for $\sqrt{s_{\text {cut }}} \sim 700 \mathrm{GeV}$, the shift in $\delta \sigma_{g g}^{\mathrm{NLO}}$ due to the last computed term in the $1 / M_{t}$ expansion is close to twelve percent. For lower values of $\sqrt{s_{\text {cut }}}$ the convergence is significantly better; for $\sqrt{s_{\text {cut }}} \sim 450 \mathrm{GeV}$, a similar shift is close to seven percent. We note that these numbers refer to NLO contributions to next-to-leading order cross sections and that the numerical significance of these shifts is further ameliorated by large leading contribution.

As we already mentioned in the Introduction, QCD corrections to Higgs boson pair production cross section at the LHC are large. To illustrate this, in the left pane of Fig. 8 we show the $K$ factor, defined as the ratio of NLO and LO $p p \rightarrow H H+X$ cross sections, evaluated at $\mu=2 m_{H}$, as a function of $\sqrt{s_{\text {cut }}}$. In general, the $K$-factors are large, confirming earlier observation of Ref. [13]. However, the $K$-factor is also strongly dependent on $s_{\text {cut }}$, decreasing significantly from the threshold region $\sqrt{s_{\text {cut }}} \approx 2 m_{H}$ to large values of $s_{\text {cut }}$. This change of the $K$-factor is related to additional suppression of the leading order cross section for $g g \rightarrow H H$ in the threshold region that we mentioned in the Introduction and the fact that for the NLO amplitude such suppression is not present. Because of that, the $K$-factor in the threshold region is enhanced. To illustrate this point, we show the partonic $g g$ cross section expanded to first non-vanishing orders in $\rho$ and 

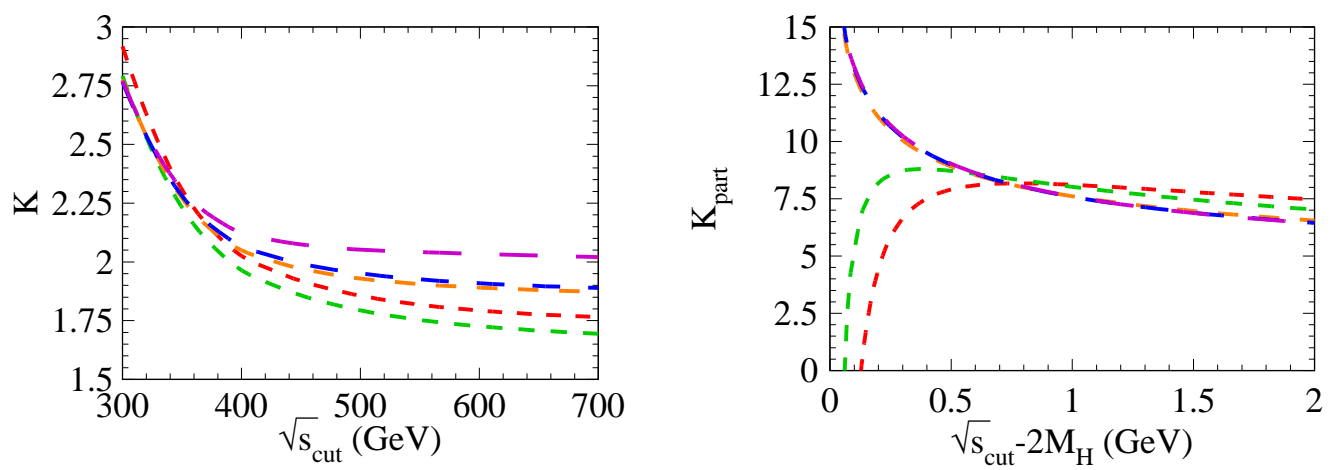

Figure 8: The NLO $K$-factors defined as the ratio of NLO and LO hadronic (left) and partonic (right) cross sections are shown as the function of $\sqrt{s_{\text {cut }}}$. The renormalization and factorization scales are chosen to be $\mu=2 m_{H}$. The color coding is as in Fig. 5

$\delta$ at leading and next-to-leading order in the threshold region 2

$$
\begin{aligned}
\sigma_{g g} & \approx \frac{G_{F}^{2} m_{H}^{2}}{2 \pi}\left(\frac{\alpha_{s}}{\pi}\right)^{2}\left\{\frac{7 \rho}{25920} \delta^{3 / 2}+\rho^{2}\left(\frac{49}{4147200} \delta^{1 / 2}+\frac{24001}{87091200} \delta^{3 / 2}\right)\right. \\
+ & \frac{\alpha_{s}}{\pi}\left[-\frac{\delta^{3 / 2}}{1296}+\rho\left(-\frac{7}{103680} \sqrt{\delta}+\delta^{3 / 2}\left\{\frac{8957}{466560}-\frac{7 \pi^{2}}{25920}+\frac{7}{1620} \ln \frac{\mu^{2}}{m_{H}^{2}}+\left(-\frac{7}{270}\right.\right.\right.\right. \\
& \left.\left.\left.-\frac{7}{2160} \ln \frac{\mu^{2}}{m_{H}^{2}}\right) \ln 2+\frac{7}{540} \ln ^{2} 2+\left(-\frac{7}{810}+\frac{7}{720} \ln 2-\frac{7}{4320} \ln \frac{\mu^{2}}{m_{H}^{2}}\right) L_{\delta}+\frac{7}{4320} L_{\delta}^{2}\right\}\right) \\
& +\rho^{2}\left(\left\{\frac{128399}{261273600}-\frac{49}{4147200} \pi^{2}+\frac{49}{345600} \ln \frac{\mu^{2}}{m_{H}^{2}}+\frac{49}{86400} \ln ^{2} 2+\frac{49}{691200} L_{\delta}^{2}\right.\right. \\
& \left.+\left(-\frac{49}{57600}-\frac{49}{345600} \ln \frac{\mu^{2}}{m_{H}^{2}}\right) \ln 2+\left(-\frac{49}{172800}-\frac{49}{691200} \ln \frac{\mu^{2}}{m_{H}^{2}}+\frac{49}{115200} \ln 2\right) L_{\delta}\right\} \delta^{1 / 2} \\
& +\left\{\frac{318919}{15676416}-\frac{24001}{87091200} \pi^{2}+\frac{193037}{43545600} \ln \frac{\mu^{2}}{m_{H}^{2}}+\frac{24001}{1814400} \ln ^{2} 2\right. \\
& +\frac{24001}{14515200} L_{\delta}^{2}+\left(-\frac{24001}{907200}-\frac{24001}{7257600} \ln \frac{\mu^{2}}{m_{H}^{2}}\right) \ln 2 \\
& \left.\left.\left.\left.+\left(-\frac{382987}{43545600}-\frac{24001}{14515200} \ln \frac{\mu^{2}}{m_{H}^{2}}+\frac{24001}{2419200} \ln 2\right) L_{\delta}\right\} \delta^{3 / 2}\right)\right]+\ldots\right\}
\end{aligned}
$$

where $L_{\delta}=\ln \delta$ and ellipses stand for terms additionally suppressed by powers of either $\rho$ or $\delta$. We observe that in the limit $\delta \rightarrow 0$, dominant contributions to $\sigma_{g g}$ come from a term $\mathcal{O}\left(\alpha_{s}^{2} \rho^{2} \sqrt{\delta}\right)$ at leading order and from a term $\mathcal{O}\left(\alpha_{s}^{3} \rho^{2} \sqrt{\delta} \ln \delta^{2}\right)$ at next-to-leading order. This implies that the behavior of the $K$-factor in the $\delta \rightarrow 0$ limit is strongly affected by power-suppressed $1 / M_{t}$ terms and that the $K$-factor becomes infinite at the exact $\delta \rightarrow 0$ threshold for $H H$ production. This point is further illustrated in the right pane of Fig. 8 where partonic $K$-factors are shown in the vicinity of the two-Higgs threshold. It follows from that plot that the threshold limit of the $\mathcal{O}\left(\rho^{0,1}\right)$ curves is significantly different from the approximation that includes $\mathcal{O}\left(\rho^{2}\right)$ and higher-

\footnotetext{
${ }^{2}$ The $\mathcal{O}\left(\rho^{0}\right)$ leading order partonic cross section scales as $\delta^{5 / 2}$ at threshold.
} 


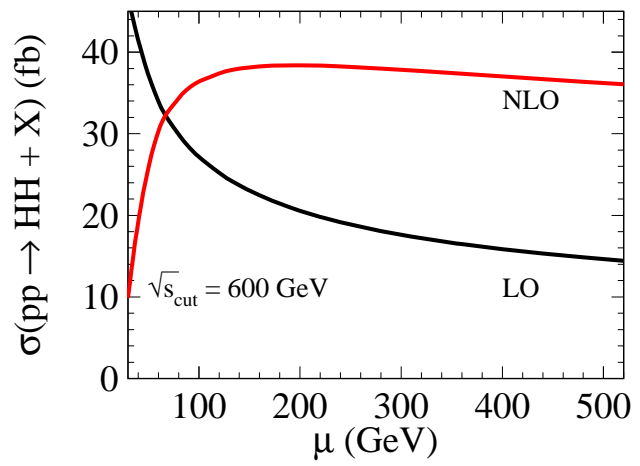

Figure 9: Scale dependence of the hadronic production cross section for $p p \rightarrow H H$.

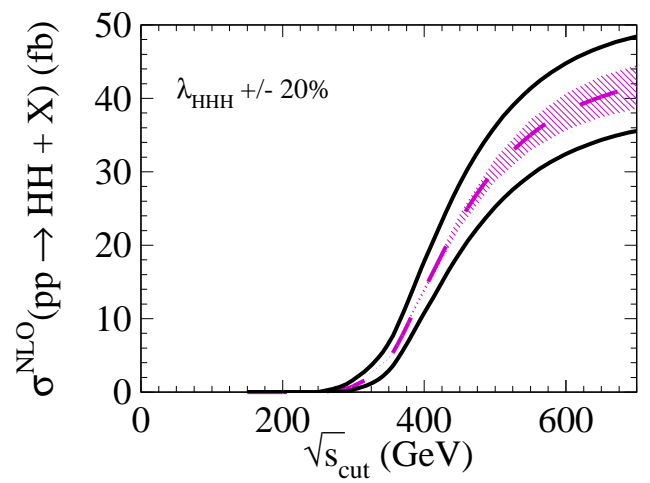

Figure 10: The NLO hadronic cross section at the $14 \mathrm{TeV}$ LHC as a function of $\sqrt{s_{\text {cut }}}$. Two black curves correspond to $\pm 20 \%$ variation in the triple Higgs boson coupling relative to its SM value. The violet (hatched) band shows the uncertainty in the SM prediction for $p p \rightarrow H H$ due to uncalculated $1 / M_{t}$ corrections.

power corrections. We also note that $1 / M_{t}$-corrections change the hadronic $K$-factor by about 14 percent at $\sqrt{s_{\text {cut }}}=700 \mathrm{GeV}$ and the change decreases for smaller values of $s_{\text {cut }}$. The shift in the $\sqrt{s_{\text {cut }}}=700 \mathrm{GeV} K$-factor due to the last computed $1 / M_{t}$ correction is close to seven percent.

In Fig. 9 we show the residual dependence of the production cross section $p p \rightarrow H H$ on the factorization and renormalization scales that we set equal to each other. The NLO cross section is computed with all available $1 / M_{t}$ corrections included. The cut on the partonic center-ofmass collision energy of $600 \mathrm{GeV}$ is imposed. It follows from Fig. 9 that the NLO QCD cross section is practically independent of the renormalization and factorization scales in a broad range of $\mu$. Choosing $\mu=2 m_{H}$ as the central value and estimating the uncertainty by increasing and decreasing $\mu$ by a factor of two, we arrive at the NLO cross section estimate $\sigma_{p p \rightarrow H H}=38_{-2}^{+0} \mathrm{fb}$ for $\sqrt{s_{\text {cut }}}=600 \mathrm{GeV}$ to be compared to $\sigma_{p p \rightarrow H H}=18_{-4}^{+6} \mathrm{fb}$ at LO. The scale-dependence uncertainty of the NLO cross section is therefore close to five percent, a significant improvement compared to $\mathcal{O}(30 \%)$ uncertainty of the leading order cross section. 
Finally, we briefly discuss implications of our results for the extraction of the triple Higgs boson coupling by comparing variations in $p p \rightarrow H H$ cross section induced by changes in $\lambda_{H H H}$ and the uncertainties in the Standard Model prediction for that cross section. In particular, we focus on the uncertainties caused by imperfect knowledge of the $1 / M_{t}$ corrections to the cross section. For our best estimate of the $p p \rightarrow H H$ cross section, we use exact leading order result and nextto-leading contributions expanded to highest known power in $\rho$ (i.e. $\rho^{4}$ for $g g$ channel and $\rho^{6}$ for $q g$ and $q \bar{q}$ channel). We assign the one-sided error to this cross section by comparing it with a similar computation that employs NLO cross sections expanded through $\rho^{3}$ in $g g$ channel and $\rho^{6}$ in $q g$ and $q \bar{q}$ channels. In Fig. 10 we show the NLO QCD cross sections for double Higgs boson production for three values of triple Higgs boson coupling, as a function of the cut on partonic center-of-mass energy. Two black curves correspond to $\pm 20 \%$ variations in the triple Higgs boson coupling; such variations cause $\mathcal{O}(20 \%)$ changes in the cross section since, as we discussed in the Introduction, the impact of the triple Higgs boson coupling on the $p p \rightarrow H H$ cross section is caused by the destructive interference of box and triangle contributions. The violet (hatched) band shows Standard Model (SM) prediction for $p p \rightarrow H H$ including the uncertainty of the $1 / M_{t}$ expansion. We conclude from Fig. 10 that the quality of our current knowledge of the Higgs boson pair production cross section, inasmuch as $1 / M_{t}$ corrections are concerned, is sufficient to detect $\mathcal{O}(10 \%)$ deviations in Higgs triple boson coupling, relative to its Standard Model value.

\section{Conclusions}

In this paper, we studied top quark mass corrections to the production cross section of the Higgs boson pair at the LHC through next-to-leading order in perturbative QCD. This is an interesting process since it allows us to directly explore the self-interaction potential of the Higgs field. QCD corrections to Higgs boson pair production are known to be very large [13]; they enhance the Higgs pair production cross section by almost a factor two. For technical reasons, these corrections were originally computed [13] in the infinite top quark mass approximation which, for realistic Higgs boson masses, has very limited applicability. For phenomenological applications, it is important to check the stability of this result against $1 / M_{t}$ power corrections. Computation of such mass corrections at next-to-leading order in QCD is the main goal of this paper.

We calculated power corrections through $\mathcal{O}\left(1 / M_{t}^{8}\right)$ for $g g \rightarrow H H$ partonic channel and through $\mathcal{O}\left(1 / M_{t}^{12}\right)$ for $q g$ and $q \bar{q}$. These corrections turned out to be large and poorly convergent which is hardly surprising since similar behavior can be observed already at leading order, where many terms in $1 / M_{t}$ expansion can be compared to the exact result. We have shown that the problem of poor convergence can be cured if the exact leading-order cross section is used to normalize the NLO QCD corrections. With such normalization, we find that mass corrections provide $\mathcal{O}(10 \%)$ increase in the NLO QCD prediction for $p p \rightarrow H H$ production cross section. 
Our computation provides a realistic estimate of the NLO QCD effects in the total cross section for a Higgs boson pair production. It justifies the use of the large- $M_{t}$ approximation to describe QCD corrections to this process and opens up a way to reliably estimate NNLO QCD corrections to $p p \rightarrow H H$ Given a very small dependence of the cross section on the renormalization and factorization scales, which may appear to be accidental given the magnitude of the NLO QCD corrections, computation of NNLO QCD corrections is important for quantifying theoretical uncertainty in $\sigma(p p \rightarrow H H)$. Moreover, it is worth remembering that observation of $p p \rightarrow H H$ is difficult and requires good control of kinematic properties of Higgs bosons decay products. It remains an important and challenging problem to extend NLO QCD results presented in this paper to describe kinematic distributions relevant for $p p \rightarrow H H$ observation.

\section{Acknowledgments}

The research of J.H, J.G. and M.S. is supported by the Deutsche Forschungsgemeinschaft in the Sonderforschungsbereich Transregio 9 "Computational Particle Physics". The research of K.M. is partially supported by US NSF under grants PHY-1214000. The research of K.M. and J.G. are partially supported by Karlsruhe Institute of Technology through is distinguished researcher fellowship program.

\section{References}

[1] G. Aad et al. [ATLAS Collaboration], Phys. Lett. B716, 1 (2012).

[2] S. Chatrchyan et al., [CMS Collaboration], Phys. Lett. B716, 30 (2012).

[3] ATLAS Collaboration, ATLAS-CONF-2013-034 .

[4] E.W.N. Glover and J.J. van der Bij, Nucl. Phys. B309, 282 (1988).

[5] T. Plehn, M.Spira and P.M. Zerwas, Nucl. Phys. B479, 46 (1996).

[6] A. Djouadi, W. Kilian, M. Mühlleitner and P.M. Zerwas, Eur. Phys. J. C10, 45 (1999).

[7] U. Baur, T. Plehn, and D. Rainwater, Phys. Rev. D69, 053004 (2004).

[8] A. Papaefstathiou, L. L. Yang and J. Zurita, Phys. Rev. D 87 (2013) 011301 arXiv:1209.1489 [hep-ph]].

[9] M. J. Dolan, C. Englert and M. Spannowsky, JHEP 1210 (2012) 112 arXiv:1206.5001 [hep$\mathrm{ph}]$.

[10] S. Dawson, Nucl. Phys. B359, 283 (1991).

\footnotetext{
${ }^{3}$ Very recently, next-to-next-to-leading order soft and virtual corrections were computed in this limit 32 .
} 
[11] A. Djouadi, M. Spira and P.M. Zerwas, Phys. Lett. B70, 1372 (1991).

[12] M. Spira, A. Djouadi, D. Graudenz and P. M. Zerwas, Nucl. Phys. B 453, 17 (1995).

[13] S. Dawson, S. Dittmaier and M. Spira, Phys. Rev. D 58, 115012 (1998) hep-ph/9805244.

[14] S. Dawson, E. Furlan and I. Lewis, Phys. Rev. D 87 (2013) 014007 arXiv:1210.6663 [hep-ph]].

[15] A.D. Martin, W.J. Stirling, R.S. Thorne and G. Watt, Eur. Phys. J. C63, 189 (2009).

[16] A. Pak, M. Rogal and M. Steinhauser, JHEP 1002, 025 (2010) arXiv:0911.4662 [hep-ph]].

[17] C. Anastasiou and K. Melnikov, Nucl. Phys. B 646, 220 (2002) hep-ph/0207004.

[18] F. V. Tkachov, Phys. Lett. B 100, 65 (1981).

[19] K. G. Chetyrkin and F. V. Tkachov, Nucl. Phys. B 192, 159 (1981).

[20] P. Nogueira, J. Comp. Phys. 105, 279 (1993).

[21] R. Harlander, T. Seidensticker and M. Steinhauser, Phys. Lett. B 426, 125 (1998); T. Seidensticker, arXiv:hep-ph/9905298.

[22] J. A. M. Vermaseren, math-ph/0010025.

[23] M. Tentyukov and J. A. M. Vermaseren, Comput. Phys. Commun. 181 (2010) 1419 hep-ph/0702279 [HEP-PH]].

[24] A. V. Smirnov, JHEP 0810 (2008) 107 arXiv:0807.3243 [hep-ph]].

[25] A. V. Smirnov and V. A. Smirnov, arXiv:1302.5885 [hep-ph].

[26] S. Laporta and E. Remiddi, Phys. Lett. B 379, 283 (1996); S. Laporta, Int. J. Mod. Phys. A 15, 5087 (2000).

[27] T. Aaltonen et al. [CDF and D0 Collaborations], Phys. Rev. D 86 (2012) 092003 arXiv:1207.1069 [hep-ex]].

[28] S. Marzani, R. D. Ball, V. Del Duca, S. Forte and A. Vicini, Nucl. Phys. B 800, 127 (2008) arXiv:0801.2544 [hep-ph]]; Nucl. Phys. Proc. Suppl. 186, 98 (2009) arXiv:0809.4934 [hep$\mathrm{ph}]$;

[29] R. V. Harlander and K. J. Ozeren, JHEP 0911, 088 (2009) arXiv:0909.3420 [hep-ph]];

[30] A. Pak, M. Rogal and M. Steinhauser, JHEP 1109 (2011) 088 arXiv:1107.3391 [hep-ph]].

[31] R. V. Harlander, H. Mantler, S. Marzani and K. J. Ozeren, Eur. Phys. J. C 66, 359 (2010) arXiv:0912.2104 [hep-ph]].

[32] D. de Florian and J. Mazzitelli, arXiv:1305.5206 [hep-ph]. 